\title{
I risultati delle prove INVALSI 2021
}

\section{Rosario Coluccia}

PUBBLICATO: 19 LUGLIO 2021

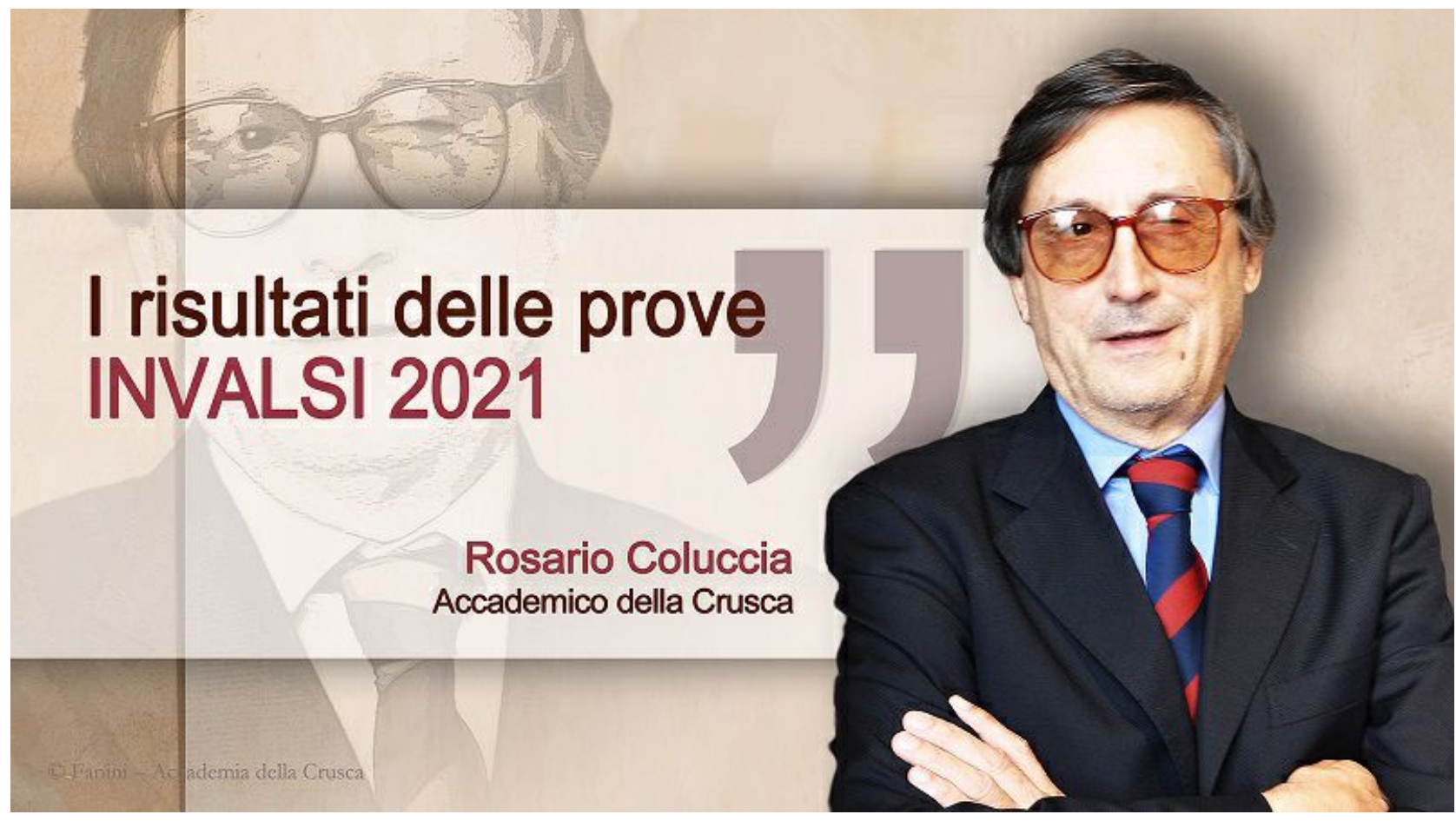

I 1 sito ufficiale di INVALSI ha da poco pubblicato i risultati delle Prove nazionali 202r. L'indagine ha coinvolto oltre I.Ioo.ooo allievi della Scuola primaria (classi II e V), circa 530.000 studenti della Secondaria di primo grado (classe III) e circa 475.0oo studenti della Secondaria di secondo grado (ultimo anno). Una campionatura estesa, volta a misurare il livello di competenza in Italiano, Matematica e Inglese raggiunto dalle diverse fasce di studenti: in sostanza fotografa lo stato di salute del nostro sistema scolastico. Le prove di quest'anno sono significative perché sono le prime dopo lo scoppio della pandemia, in un anno scolastico caratterizzato dalla coesistenza di didattica in presenza e didattica a distanza (alternanti a seconda delle situazioni e delle variabili condizioni sanitarie). Eccone di seguito i risultati, riassunti in maniera molto sintetica.

Nella scuola primaria il confronto dell'indagine 202I con la precedente 2019 presenta un quadro di sostanziale stabilità. I risultati medi per Italiano sono oggi in tutto il Paese simili a quelli dell'indagine precedente, con un leggero incremento degli allievi che si attestano ai livelli più alti. Per Matematica si osserva un leggero calo del risultato medio complessivo rispetto al 2019 e una piccola riduzione del numero degli allievi che raggiungono risultati buoni o molto buoni. Complessivamente buoni e non dissimili da quelli del 2019 sono i risultati per Inglese.

È meno rassicurante la situazione della scuola secondaria di primo grado. Rispetto agli anni precedenti, non raggiunge livelli adeguati di competenza il 39\% degli studenti per Italiano (5 punti percentuali in più sia rispetto al 2018 sia rispetto al 2019); il $45 \%$ degli studenti per Matematica (5 punti percentuali in più rispetto al 2018 e 6 punti percentuali in più rispetto al 2019); il 24\% degli studenti per Inglese-lettura (2 punti percentuali in meno rispetto al 2018 e 2 punti percentuali in più 
rispetto al 20I9), il 4I\% per Inglese-ascolto (3 punti percentuali in meno rispetto al 2018 e I punto percentuale in più rispetto al 2019).

Per quanto riguarda la scuola secondaria di secondo grado, i risultati del 202I (paragonati a quelli del 20I9) sono più bassi in Italiano e in Matematica, modeste le variazioni in Inglese (sia lettura sia ascolto). Non raggiunge livelli soddisfacenti il $44 \%$ degli studenti per Italiano (9 punti percentuali in più rispetto al 2019), il $51 \%$ per Matematica (9 punti percentuali in più rispetto al 2019), il $51 \%$ per Inglese-lettura (3 punti percentuali in più rispetto al 2019), il 63\% per Inglese-ascolto (2 punti percentuali in più rispetto al 2019). Il calo è vistoso per Italiano e Matematica, più contenuto, con oscillazioni poco più che fisiologiche, per Inglese.

Un elemento accomuna i diversi ordini di scuola e richiede particolare attenzione. I divari territoriali tendono ad ampliarsi. Se, a mo' d'esempio, ci riferiamo alla scuola media in alcune regioni del Mezzogiorno si riscontra un maggior numero di allievi con livelli di competenza molto bassi, che raggiunge il 50\% e oltre della popolazione scolastica in Italiano, il 60\% in Matematica, il 30-40\% in Inglese-lettura e il 55-60\% in Inglese-ascolto. In generale gli studenti del Nord e del Centro ottengono valutazioni migliori rispetto a quelli del Sud (se ci riferiamo alle verifiche sostanziali, non ai voti raggiunti alla fine dell'anno scolastico, i criteri di giudizio sono diversi). In tutte le materie le perdite maggiori di apprendimento si registrano tra gli allievi che provengono da contesti socio-economicoculturali sfavorevoli. Parallelamente, tra questi ultimi diminuisce la quota di studenti con risultati elevati.

La diffusione dei risultati INVALSI ha provocato resoconti allarmati sui giornali. Eccone un paio, scelti a caso: "La Dad ha fatto crac. [...] Il referto delle prove Invalsi non lascia scampo: resistono solo le elementari, ma dalle medie in su è una Caporetto" (CdS I5 luglio); "Perdite negli apprendimenti in tutto il Paese: la Dad è stato uno strumento d'emergenza che non poteva risolvere tutti i problemi" (La Stampa, I6 luglio).

Proviamo a riflettere, sul passato e su quello che potrebbe aspettarci. Non c'è dubbio che la scuola "vera" sia in presenza, per ragioni didattiche e anche per esigenze di socializzazione e di scambio di esperienze. La relazione tra compagni, il rapporto tra discente e docente, la condivisione di esperienze hanno riflessi positivi sull'apprendimento. Ma la didattica a distanza non può essere demonizzata: imposta dalla pandemia, è stato l'unico mezzo che ha consentito di fare scuola in un lungo periodo di difficoltà estrema. Ovviamente la soluzione di emergenza non poteva risolvere problemi preesistenti. Al contrario: il contesto difficile ha reso più evidenti le precedenti insufficienze educative e i divari territoriali della nostra Nazione.

PISA è l'acronimo di "Programme for International Student Assessment", indagine internazionale con cadenza triennale promossa dall'OCSE, con l'obiettivo di rilevare le competenze degli studenti di i5 anni in Lettura (capacità di leggere e comprendere un testo, possesso della lingua nazionale), Matematica e Scienze. Il primo ciclo dell'indagine si è svolto nel 2000; i risultati del 2018 (anno prepandemico) sono stati pubblicati nel dicembre 2019. Alla rilevazione PISA 2018 hanno partecipato 79 paesi; per l'Italia II.785 studenti quindicenni, provenienti da 550 scuole. Nella prova di Lettura il punteggio medio ottenuto dagli studenti italiani è leggermente più basso rispetto a quello della media OCSE; nella prova di Matematica è in linea con la media OCSE, nella prova di Scienze è più basso rispetto alla media OCSE. In un arco temporale più ampio, il livello di preparazione in Lettura e in Scienze è in calo rispetto al 20r2, in Matematica il punteggio delle ultime edizioni è rimasto perlopiù stabile dallo stesso 20I2. I divari territoriali sono molto ampi, gli studenti del Nord ottengono i risultati migliori, mentre i loro coetanei del Sud sono in difficoltà: il minimo è nella zona Sud e Isole, 
il massimo nel Nord Est.

Senza dubbio, la questione della scuola non nasce oggi. Tutto si svolge nell'indifferenza pressoché generale. Di fronte all'emergenza COVID-I9, i cosiddetti gruppi dirigenti italiani si sono mostrati nel complesso inadeguati, agendo in maniera desultoria e spesso contraddittoria: caos totale, disorientamento generale. Nulla o pochissimo si è fatto per quanto riguarda il miglioramento degli edifici scolastici, dei trasporti, della rete digitale. Come potevano ragazzi poveri, con computer a volte obsoleti e spesso da condividere con fratelli e sorelle, avvalersi in misura soddisfacente della didattica a distanza?

$\mathrm{Ci}$ auguriamo tutti che questi siano discorsi che riguardano solo il passato. Ma forse le prospettive per la ripresa scolastica autunnale non sono rosee. Il COVID fa (giustamente) paura, anche se non suscita alcun timore quando dobbiamo festeggiare le vittorie agli europei di calcio. Cosa fare per salvaguardare la salute collettiva, se in autunno si ripresenterà la necessità della didattica a distanza? I politici facciano la loro parte, in vari campi, torno a dire. In primo luogo diano vita a un piano d'investimenti per dotare ogni alunno di un tablet o di un pe portatile, con una connessione efficiente in tutte le zone d'Italia. Verrebbe ridotto lo storico divario sociale e territoriale del nostro Paese e risulterebbero attenuate le differenze economiche e culturali di partenza, aumentate invece durante la pandemia, in contrasto con l'obiettivo costituzionale di una buona istruzione scolastica garantita a tutti.

Non sappiamo quasi nulla di quello che davvero è successo nella scuola italiana in quest'ultimo anno e mezzo, ignoriamo come professori e studenti si sono misurati con una didattica che richiede modalità diverse rispetto alle tradizionali. Di fatto, la necessità ha giocoforza comportato un aumento indotto delle competenze digitali dei docenti e anche un diverso rapporto degli studenti con pc e tablet, finora dagli stessi utilizzati prevalentemente con finalità molto diverse. Con cautela possiamo affermare che, almeno, la situazione generale impone una riflessione collettiva su modalità, limiti e pregi della didattica a distanza. Una presa di coscienza è necessaria, non ha senso discutere se il cavallo è più veloce rispetto alla locomotiva a vapore, devono coesistere entrambi.

Chiunque è a contatto con il mondo della scuola sa che anche quest'anno molti professori hanno lavorato in maniera eccellente, superando difficoltà inedite, coinvolgendo gli studenti, ottenendo risultati ottimi. Chi vuole trova esempi concreti in rete, dove resoconti, pdf e power point testimoniano l'ottima attività svolta nelle classi (potrei indicare alcuni siti, ma evito favoritismi, sia pure involontari). Con atteggiamento propositivo, molti professori hanno sfruttato le potenzialità del digitale; e gli studenti hanno partecipato attivamente. È questa la via da seguire, se la situazione sanitaria richiederà ancora forme di didattica a distanza. E anche se non fossero strettamente necessarie. Non per rassegnazione. La scuola italiana è chiamata a compiti straordinari, tradizionale e nuovo devono convivere. La sfida va accettata.

\section{Cita come:}

Rosario Coluccia, I risultati delle prove INVALSI 2021, "Italiano digitale", XVIII, 2021/3 (lugliosettembre)

DOI: $10.35948 / 2532-9006 / 2021.9585$

Copyright 2021 Accademia della Crusca

Pubblicato con licenza creative commons CC BY-NC-ND 